\title{
Assessment of pancreatic duct damage following trauma: is endoscopic retrograde cholangiopancreatography the gold standard?
}

\author{
N Doctor, JS Dooley, BR Davidson
}

\begin{abstract}
Summary
A 12-year-old girl was admitted as an emergency with blunt pancreatic trauma. Computed tomography (CT) showed an intact pancreas. She failed conservative treatment. Endoscopic retrograde cholangiopancreatography (ERCP) demonstrated division of the pancreatic duct at the neck of pancreas. At laparotomy, however, there was complete necrosis of the pancreatic head. She recovered well following enteric drainage of the body of pancreas. CT and ERCP are considered the optimal imaging for pancreatic trauma but the findings may be misleading.
\end{abstract}

Keywords: pancreatic trauma, pancreatic duct, endoscopic retrograde cholangiopancreatography

\section{Introduction}

Severe pancreatic trauma is associated with a mortality of $10-20 \%$, although this figure may be reduced by careful assessment of the injury and appropriate surgery. ${ }^{1}$ Endoscopic retrograde cholangiopancreatography (ERCP) has been suggested as the definitive method of diagnosing pancreatic duct damage and planning surgery. ${ }^{2}$ We report a case in which ERCP findings did not correlate with those at surgery.

\section{Case report}

A 12-year-old girl was transferred to our unit $30 \mathrm{~h}$ after being kicked in the epigastrium by a horse. Initial assessment at the referring hospital revealed upper abdominal bruising and tenderness. An abdominal computed tomography (CT) scan showed an oedematous pancreas and she was referred for further management. Following transfer she complained of continuing severe upper abdominal pain. She was haemodynamically stable with localised upper abdominal signs of tenderness and mild guarding. Blood investigations revealed haemoglobin $12.3 \mathrm{~g} / \mathrm{dl}(11.5-15.5)$, white cell count $20.5 \times 10^{9} / 1(4.5-11.0)$ and platelets $321 \times 10^{9} / 1$ (140-400) (normal ranges). Her amylase was $90 \mathrm{IU} / 1(70-220)$; urea and electrolytes, liver function tests and clotting screen were normal.

She was treated conservatively with nil by mouth, intravenous fluids and an octreotide (Sandoz, UK) infusion $(50 \mu \mathrm{g}$ tid intravenously). A CT scan with intravenous bolus contrast enhancement was repeated after $72 \mathrm{~h}$ which again showed an oedematous pancreas with no focal necrosis. Her condition did not improve with further conservative treatment and an ERCP was therefore performed. This revealed an intact duct in the head of the pancreas with a leak of contrast at the neck suggestive of a neck transection (figure 1).

At laparotomy the head of pancreas was completely necrotic and liquified. However, the duodenum and the body and tail of the pancreas were intact and appeared well perfused, non-oedematous and healthy. After removing the necrotic material from the region of the head of pancreas the duct in the body of the pancreas was identified and anastomosed to a Roux loop of jejunum. The patient made a complete clinical recovery and was discharged from hospital three weeks post operatively.

\section{Discussion}

Blunt trauma to the pancreas is an uncommon injury and therefore experience of its assessment and management is limited even in the major centres. ${ }^{3}$ Rapid intravenous bolus enhanced CT scanning has proven effective in the assessment of pancreatic necrosis following

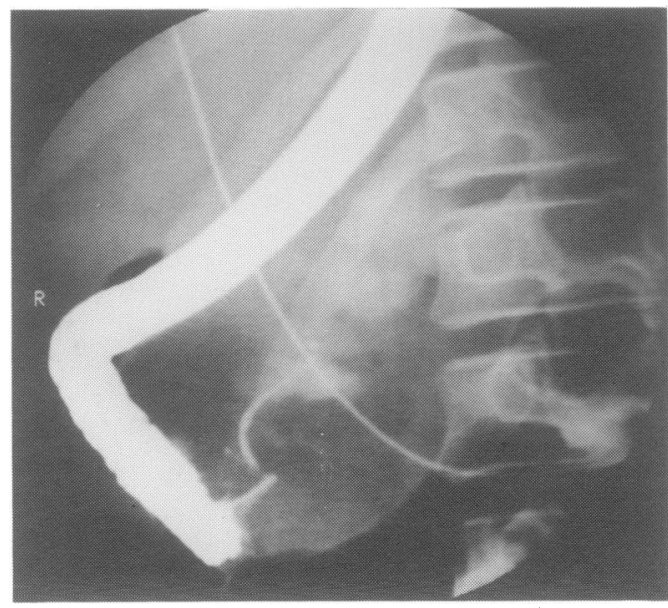

Figure 1 Endoscopic retrograde cholangiopancreatography demonstrating a normal pancreatic duct within the head of pancreas and a leak from the neck of pancreas. 


\begin{tabular}{|l|}
\hline Learning points \\
\hline - ERCP is of value in the diagnosis of duct \\
transection \\
- Complete pancreatic necrosis may occur \\
leaving the duct intact \\
\hline
\end{tabular}

acute pancreatitis. ${ }^{4}$ However Sivit and colleagues suggested that this method was unreliable for assessing blunt pancreatic trauma in children as was our experience in the present case. ${ }^{5}$ This discrepancy may be due to the evolution of the pancreatic necrosis in relation to the timing of the imaging and subsequent surgery.

One of the major clinical questions in panc-

1 Wilson RH, Moorehead RJ. Current management of trauma to the pancreas. Br $\mathcal{f}$ Surg 1991; 78: 1196-202.

2 Hayward S, Lucas CE, Sugava C, Ledgerwood A Emergency ERCP: a highly specific test for acute pancreatic trauma. Arch Surg 1989; 124: 745-6.

3 Wisner DH, Wold RL, Frey CF. Diagnosis and treatment of pancreatic injuries. Arch Surg 1990; 125: 1109-13.

4 London NJM, Leese T, Lavelle JM, et al. Rapid bolus contrast enhanced dynamic computed tomography in acute pancreatitis: a prospective study. Br $¥$ Surg 1991; 78: 1452-6. reatic trauma is whether the injury has produced duct transection and some authors have suggested that decisions on the necessity for surgery and its nature can be guided by ERCP findings. ${ }^{2}$ This case has clearly shown that ERCP findings may also be misleading and that an area of complete pancreatic necrosis may occur whilst the duct crossing that segment of pancreas appears intact. Since the pancreatic duct is prone to division in severe pancreatic trauma, ${ }^{6}$ this situation may be due to the pancreatic duct's resilience to digestion by activated pancreatic enzymes.

We would conclude that ERCP is of value in the diagnosis of duct transection but that an intact duct at ERCP does not relate to pancreatic viability or the surgical procedure necessary in treatment.

5 Sivit CJ, Eichelberger MR, Taylor GA, Bulas DI, Gotschall CS, Kushner DC. Blunt pancreatic trauma in children: CT diagnosis. AFR 1992; 158: 1097-100.

6 Frey CF, Wardell JW. Injuries to the pancreas. In: Trede M Carter DC, Surgery of the pancreas. London: Churchil Livingstone, 1993, pp 565-89.

7 Neoptolemos JP, London NJM, Carr-Locke DL. Assessment of main pancreatic duct integrity by endoscopic retrograde pancreatography in patients with acute pancreatitis. Br F Surg 1993; 80: 94-9.

\title{
Endocarditis due to high-level gentamicin- resistant Enterococcus faecalis
}

\author{
PYC Lee, SS Das
}

\begin{abstract}
Summary
We report a case of aortic valve endocarditis caused by Enterococcus faecalis highly resistant to gentamicin, which failed to respond to conventional antibiotic combination therapy. Extensive in vitro testing was required to determine an appropriate antimicrobial regimen. Despite bacteriological resolution and cardiac surgery the patient died from complications of infective endocarditis.
\end{abstract}

Keywords: Enterococcus faecalis, endocarditis

Public Health

Laboratory, St

George's Hospital,

London SW17 0QT,

UK

P YC Lee

SS Das

Correspondence to

Dr PYC Lee, Public Health

Laboratory, St George's

Hospital, Blackshaw Road,

London SW 17 0QT, UK

Accepted 21 September 1994

\section{Introduction}

Enterococci are intrinsically relatively less suspectible to penicillin and other cell-wallactive agents, including vancomycin and teicoplanin. ${ }^{1}$ They are also intrinsically relatively resistant to aminoglycosides. However, the combination of penicillin (or vancomycin) with an aminglycoside achieves a synergistic bactericidal effect, and is the established antibiotic regimen for the successful treatment of enterococcal endocarditis. The first case of endocarditis caused by a high-level gentamicin-resistant (HLGR) enterococcus was reported in 1984 from the US. ${ }^{2}$ Since then, nine such cases, including two in the UK, have been reported and recently reviewed by Moellering. ${ }^{3}$ This is only the third reported case of endocarditis caused by a HLGR Enterococcus faecalis in the UK.

\section{Case report}

A 40-year-old man was admitted with a twomonth history of fever, night sweats, rigors and a painful leg. On examination, he had a fever of $38.6^{\circ} \mathrm{C}$, signs of aortic regurgitation, finger clubbing, anaemia, two scrotal abscesses and deep vein thromboses in both calves clinically. Echocardiography confirmed aortic regurgitation and detected vegetations on the aortic valve. The clinical diagnosis of infective endocarditis was confirmed with the isolation of $E$ faecalis in five sets of blood cultures. Treatment was initiated with intravenous benzylpenicillin ( 2 mega-units every four hours) and gentamicin (dosage determined by serum levels). Ciprofloxacin ( $400 \mathrm{mg}$ twice daily) intravenously was substituted for gentamicin when HLGR was detected. Pus from the scrotal abscesses grew the same organism. Despite initial resolution of fever with negative 